\title{
Cryptanalysis in Prime Order Subgroups of $Z_{n}^{*}$
}

\author{
Wenbo $\mathrm{Mao}^{1}$ and Chae Hoon $\mathrm{Lim}^{2}$ \\ 1 Hewlett-Packard Laboratories, \\ Filton Road, Stoke Gifford, Bristol BS12 6QZ, United Kingdom \\ wm@hplb.hpl.hp.com \\ 2 Future Systems Inc., \\ 372-2, Yang Jae-Dong, Seo Cho-Gu, Seoul, 137-130, Korea \\ chlim@future.co.kr
}

\begin{abstract}
Many cryptographic protocols and cryptosystems have been proposed to make use of prime order subgroups of $Z_{n}^{*}$ where $n$ is the product of two large distinct primes. In this paper we analyze a number of such schemes. While these schemes were proposed to utilize the difficulty of factoring large integers or that of finding related hidden information (e.g., the order of the group $Z_{n}^{*}$ ), our analyzes reveal much easier problems as their real security bases. We itemize three classes of security failures and formulate a simple algorithm for factoring $n$ with a disclosed non-trivial factor of $\phi(n)$ where the disclosure is for making use of a prime order subgroup in $Z_{n}^{*}$. The time complexity of our algorithm is $O\left(n^{1 / 4} / f\right)$ where $f$ is a disclosed subgroup order. To factor such $n$ of length up to 800 bits with the subgroup having a secure size against computing discrete logarithm, the new algorithm will have a feasible running time on use of a trivial size of storage.
\end{abstract}

\section{Introduction}

Let $n=p q$ where $p$ and $q$ are large primes. The multiplicative group of integers modulo $n$, which we denote $Z_{n}^{*}$, has a secret order (the number of elements in the group) $(p-1)(q-1)$. It is assumed to be difficult to discover this quantity from $n$, and the difficulty has been used as the security basis for many cryptosystems and protocols including RSA [16], Fiat-Shamir [7], Rabin [15], Guillou-Quisquater [10], and many many more.

In the literature we also often see cryptosystems and cryptographic protocols (crypto schemes) that make use of prime order subgroups of $Z_{n}^{*}$ (e.g., $\left[\begin{array}{lllll}112 & 8 & 9 & 11 & 13\end{array}\right]$ ). In the sequel, whenever we say subgroup, we refer to a prime order subgroup of $Z_{n}^{*}$. The order of such a subgroup is a prime number. The schemes referred above involve various ways of using such subgroups. In some use (a cryptosystem [2]), subgroup elements are disclosed while their order is hidden, and the security basis is an assumed difficulty to find the order of a given element. In another use ([18911]13]), a subgroup is made public by disclosing both the order and the elements. This use allows zero-knowledge proof of some properties (such as possession or equality) of the discrete logarithms of the group elements. 
We will analyze each of the crypto schemes referred in the above paragraph and itemize three classes of security failures from our analysis. Class 1: given elements of a prime order subgroup, the group order, even as a secret, cannot be used as an RSA-like hidden trapdoor. Class 2: A disclosed prime order subgroup allows to solve problems which should be difficult in $Z_{n}^{*}$ had the subgroup not been disclosed. Class 3: the size of a disclosed subgroup versus that of $Z_{n}^{*}$ leads to a significant reduction on the complexity for factoring $n$. We will formulate a simple algorithm for factoring $n$ with a special structure designed for making use of prime order subgroups in $Z_{n}^{*}$. To factor such $n$ of length up to 800 bits, our algorithm will have a feasible running time on use of a trivial size of storage.

Throughout the paper we stipulate $n=p q$ for $p$ and $q$ being distinct large primes. For an element $a \in Z_{n}^{*}$, we will use $\operatorname{ord}_{n}(a)$ to denote the order of $a$ modulo $n$, which is the least positive integer $b$ satisfying

$$
a^{b} \equiv 1(\bmod n)
$$

We also confine ourselves to study subgroups of odd prime orders; namely, we exclude the case of order 2. Such subgroups merely contain elements which can be used to factor $n$ (if they are not trivial numbers $n-1$ or 1 ).

\section{Class 1: Absence of RSA-like Hidden Trapdoor in any Prime Order Subgroup}

Let $g \neq 1$ be a non secret element in a prime order subgroup. Since $g \bmod p$ $(g \bmod q)$ is an element in $Z_{p}^{*}\left(Z_{q}^{*}\right)$, we have the following

$$
\operatorname{ord}_{p}(g)\left|p-1, \quad \operatorname{ord}_{q}(g)\right| q-1 .
$$

So

$$
p=\operatorname{ord}_{p}(g) k+1, \quad q=\operatorname{ord}_{q}(g) \ell+1,
$$

for some (even) numbers $k$ and $\ell$. A basic fact in number theory states the following: for every $x \in Z_{n}^{*}$,

$$
m \mid n \text { implies } \operatorname{ord}_{m}(x) \mid \operatorname{ord}_{n}(x) \text {. }
$$

Since $\operatorname{ord}_{n}(g)$ is prime, from (2) we know either $\operatorname{ord}_{p}(g)=\operatorname{ord}_{n}(g)$, or $\operatorname{ord}_{p}(g)=$ 1. Same true for $\operatorname{ord}_{q}(g)$. Obviously we do not consider the case $\operatorname{ord}_{n}(g)=1$. Thus (1) consists of one of the following three cases:

$$
p=\operatorname{ord}_{n}(g) k+1, \quad q=\operatorname{ord}_{n}(g) \ell+1,
$$

or

$$
p=\operatorname{ord}_{n}(g) k+1, \quad q=\ell+1, \quad \text { with } \operatorname{ord}_{n}(g) \backslash \ell,
$$

or

$$
p=k+1, \quad q=\operatorname{ord}_{n}(g) \ell+1, \quad \text { with } \operatorname{ord}_{n}(g) \backslash k \text {. }
$$


Note that the case $(4)$ implies $g \equiv 1(\bmod q)$, or $q \mid g-1$. Noting further $0<g-1<n$, we will have (let $\operatorname{gcd}(x, y)$ denote the greatest common divisor of $x$ and $y$ )

$$
\operatorname{gcd}(g-1, n)=q \text {. }
$$

Similarly for the case (5), we will have

$$
\operatorname{gcd}(g-1, n)=p .
$$

We conclude to this end the following statement.

Proposition 1. Let $g \in Z_{n}^{*}$ be a non secret element and $\operatorname{ord}_{n}(g)$ be an odd prime. Then $n=p q$ must only use $p$ and $q$ with the structures shown in (3), or else the factorization of $n$ will be disclosed.

A previous cryptosystem [2] contained the above failure for using moduli with the construction (4). The failure was later discovered by Henk Meijer [3], with a suggestion for fixing by using moduli with the construction (3). Below we will examine that construction as per the system of [2], and in Section 4 we will further examine the same construction for a different danger.

\subsection{The Case $n=p q=\left(\operatorname{ord}_{n}(g) k+1\right)\left(\operatorname{ord}_{n}(g) \ell+1\right)$}

Further examining (3) we can see

$$
n=p q=\operatorname{ord}_{n}(g)\left(\operatorname{ord}_{n}(g) k \ell+k+\ell\right)+1 .
$$

So $n-1$ is a multiple of $\operatorname{ord}_{n}(g)$. The number $n-1$ is publicly available even $\operatorname{ord}_{n}(g)$ is kept as a secret. This fact tells us that the subgroup generated from $g$ does not have an RSA-like trapdoor. By an RSA-like trapdoor of a group, we mean a secret number which is the inverse of a public exponent modulo the group order. The trapdoor is hidden because the group order is.

Now in the case $n-1$ being a multiple of the group order, for any public exponent $e$, one can compute $d$ to satisfy

$$
e d=1(\bmod n-1)
$$

The existence of $d$ is not a problem. If $\operatorname{gcd}(e, n-1)>1$, we can replace $n-1$ in (6) with $\frac{n-1}{\operatorname{gcd}(e, n-1)}$ which should still be a multiple of $\operatorname{ord}_{n}(g)$ unless $\operatorname{gcd}(e, n-1)=$ $\operatorname{ord}_{n}(g)$ which is the case mostly welcome because we have discovered the hidden order.

Since $\operatorname{ord}_{n}(g) \mid n-1,(6)$ implies $e d=1\left(\bmod \operatorname{ord}_{n}(g)\right)$. Thus, in any subgroup of order $\operatorname{ord}_{n}(g)$, RSA-like encryption or signature algorithms will no longer be secure. Any variations relying on the secrecy of $\operatorname{ord}_{n}(g)$ will fail too. Below we review a cryptosystem that fails in this way. 


\subsection{Scheme Failure}

The scheme [23] consists of a public-key encryption algorithm and digital signature algorithm. Both work in a prime order subgroup of $Z_{n}^{*}$. The order of the subgroup is $r=\operatorname{ord}_{n}(g)$, a prime of size 160 bits which is kept secret by the owner of $n$.

In the encryption algorithm, the public key is $(g, n)$, and the private key is a number $z$ computed as follows

$$
z=-\frac{1}{2} \bmod r
$$

To encrypt a message $0<m<n$, the sender picks a random number $t$ of size less than 160 bits, and compute the ciphertext pair $(u, v)$ as follows

$$
\begin{gathered}
u:=g^{2 t} \bmod n, \\
v:=m g^{t} \bmod n .
\end{gathered}
$$

The recipient decrypts the pair $(u, v)$ by the following calculation.

$$
m:=u^{z} v \bmod n \text {. }
$$

The number $n-1$ will suffice for a non-recipient to compute $u^{z}$ and thereby decrypt $(u, v)$. Let

$$
s:=\frac{n-1}{2^{t}}
$$

for some $t$ such that $s$ is odd. A non-recipient can compute

$$
z^{\prime}=-\frac{1}{2} \bmod s
$$

Noting that $s$ is a multiple of $r$ and the latter is the order of $u$, it is easy to see

$$
u^{z^{\prime}}=u^{z} \bmod n \text {. }
$$

This means decryption can be performed by anybody.

A similar failure in the digital signature algorithm of this cryptosystem can be demonstrated analogously, using $n-1$ as the trapdoor needed. The failure allows anybody to issue signatures for the owner of the public key $(g, n)$.

\section{Class 2: Difficult Problem Made Easy Due to Disclosure of a Subgroup}

Given elements in a group, zero-knowledge proofs showing some properties regarding their discrete logarithms require to make the group order public (at least to the parties involved in the zero-knowledge protocols). But when the group in question is $Z_{n}^{*}$, the order $(p-1)(q-1)$ cannot be disclosed or else no use of the 
integer factorization problem can be made. A clever idea to solve this contradiction is to define zero-knowledge protocols working in a prime order subgroup of $Z_{n}^{*}$, with the order disclosed without leading to discovery of $(p-1)(q-1)$. A widely adopted method [1 8,9 11 13 ) to achieve this is to construct $p$ and $q$ with the structures given in (3), and make $g$ and $\operatorname{ord}_{n}(g)$ public. Here $g$ is chosen such that $\operatorname{ord}_{n}(g)=\operatorname{ord}_{p}(g)=\operatorname{ord}_{q}(g)$.

A scheme failure demonstrated here is a result of transforming a problem which is known to be difficult in $Z_{n}^{*}$, into an easy one in a prime order subgroup.

This is a group signature scheme 13. (In order to avoid confusion between a mathematical group and a group of people, in the sequel we use the bold font to refer to the latter.) A group signature scheme allows an individual member in a group (e.g., a corporation environment) to issue a signature on behalf of the group with the signer's identity hidden from the signature verifier (who verifies a signature using the public key of the group). Such signature algorithms are probabilistic ones in that, it should be computationally infeasible to decide if two signatures have been issued by the same group member. To prevent anonymity misuse, a group manager, upon inputting an administration secret and a signature, can deterministically identify the signer who has issued the signature. This is usually achieved by encrypting the signer's identity under the public key of the group manager in the time of signature issuance; the manager need not stay on line.

In this scheme the group manager's public-key cryptosystem is the ElGamal cryptosystem [6] working in a prime order subgroup of $Z_{n}^{*}$. Using the notation of [13, the subgroup is setup as follows. Let $n=p_{1} p_{2}$ where $p_{1}, p_{2}$ are distinct large primes. Let further $q$ be another prime of size about $160 \mathrm{bits}$, and $q \mid p_{1}-1$, $q \mid p_{2}-1$. Fixing an element $g \in Z_{n}^{*}$ of order $q$ (modulo both $p_{1}$ and $p_{2}$ ), the manager's public key is $y$ computed as follows

$$
y:=g^{x} \bmod n,
$$

where $x<q$ is the private key of the manager (the above-mentioned administration secret). It is assumed that $n$ and $g$ are generated by a trusted center, and nobody else, even not the group manager or members, will know the factorization of $n$. The center also generates an RSA public exponent $e$, again with nobody else knowing the inverse of $e$ modulo $(p-1)(q-1)$.

Let the group have $k$ members. Each member $i(1 \leq i \leq k)$ chooses a secret $s_{i} \in Z_{n}^{*}$ and generates an identity by encrypting the secret $s_{i}$ in RSA encryption

$$
i d_{i}:=s_{i}^{e} \bmod n
$$

From the assumption in key generation, we know that even the manager cannot learn $s_{i}$, the secrets of the members. So (s)he cannot frame any member (provided each member $(i)$ make sure not to choose $s_{i}$ of order $q$, and this is easy to check against by checking $\left.s_{i}^{q} \neq 1 \bmod n\right)$. These $k$ identities $i d_{j}(1 \leq j \leq k)$ are announced to the public, together with $g, e, n$. So the public key of the group is the following tuple

$$
\left(i d_{1}, i d_{2}, \cdots, i d_{k}, g, e, n\right)
$$


Omitting details, when the member $i$ issuing a signature, (s)he shall use the identities of all members in the group and create $k$ pairs of ElGamal-like ciphertext blocks

$$
\left(A_{j}, B_{j}\right)=\left(g^{w_{j}} \bmod n,\left(\frac{i d_{i}}{i d_{j}}\right)^{d_{j}} y^{w_{j} e} \bmod n\right), 1 \leq j \leq k,
$$

where $d_{j}$ are part of the signature value, and $w_{j}$ are random numbers chosen by the signer $i$ (for $j=1,2, \cdots, k$ ). The scheme also requires the signer to prove, in zero-knowledge, possession of the eth root of one of the $k$ identities. It is thus obvious that only a group member is able to have generated the above ciphertext pairs, and hence to have issued a signature.

At first glance, a verifier, apart from knowing the fact that each pair $\left(A_{j}, B_{j}\right)$ is generated via using the identity $i d_{j}$ (for $1 \leq j \leq k$ ), cannot identify the signer $i$ from these pairs. On the other hand, the group manager can identify the signer because each pair $\left(A_{j}^{e}, B_{j}\right)(1 \leq j \leq k)$ provides ElGamal encryption of $\left(\frac{i d_{i}}{i d_{j}}\right)^{d_{j}}$ under the manager's public key $y$, and the member $i$ is identified because decrypting $\left(A_{i}^{e}, B_{i}\right)$ will return 1 .

However we notice that 1 is an element in any subgroup of $Z_{n}^{*}$, in particular in all subgroups of order $q$. Thus,

$$
\operatorname{ord}_{n}\left(B_{i}\right)=\operatorname{ord}_{n}\left(y^{w_{j} e}\right)=q
$$

while

$$
\operatorname{ord}_{n}\left(B_{j}\right)=\operatorname{ord}_{n}\left(\left(\frac{i d_{i}}{i d_{j}}\right)^{d_{j}} y^{w_{j} e}\right) \neq q, \text { for } j \neq i
$$

These facts can easily be learned by anybody via checking whether $B_{j}^{q} \bmod n$ is 1 , for $1 \leq j \leq k$. There is no need for an outsider to find the $e$-th root in $Z_{n}^{*}$ which is well known to be a difficult problem.

A similar failure occurred in a protocol for fair-exchange of signed documents [1] (discovered by Colin Boyd [4]) which allows decryption by any non-recipient using the order of a subgroup disclosed.

The moral of this failure is that when a prime order subgroup of $Z_{n}^{*}$ is made public, great care must be taken in protocol design not to transform a problem in $Z_{n}^{*}$, which is thought to be difficult, into an easy job in the subgroup.

\section{Class 3: Significant Complexity Reduction for Integer Factorization}

Let us now examine the structure of a composite integer $n=p q$ in which a factor of $\phi(n)=(p-1)(q-1)$ is made public. The widely adopted method for disclosing a subgroup of $Z_{n}^{*}[18,911,13]$ ) is in such a structure. Let

$$
n=p q, p=2 p^{\prime} f+1, q=2 q^{\prime} f+1,
$$


where $p, q, f$ are distinct primes and $p^{\prime}, q^{\prime}$ are relatively prime integers. Here we see that the quantity

$$
r=4 f^{2}
$$

is a factor of $\phi(n)$, and in the schemes referred above, the factor $r$ is made public. (In 11], an additional factor, which is a factor of $p^{\prime}$, is also made public. We will examine that case in a moment.)

We first note

$$
n+1=\phi(n)+p+q
$$

So when $r \mid \phi(n)$ is disclosed, we have

$$
p+q \equiv n+1(\bmod r)
$$

For $r<p+q$ (otherwise the above congruence is an equation and $p+q$ is disclosed directly), we can rewrite the congruence (10) as

$$
p+q=k r+(n+1 \bmod r),
$$

where $k$ is a unknown quantity to be determined. From (11) it is easy to see $|k| \approx|p+q|-|r|$, where $|a|$ denotes the bit length of the integer $a$ in the binary representation. Notice that if $p+q$ becomes known, factoring $n$ follows a simple calculation. For known $r$, finding $p+q$ using (11) is equivalent to finding the unknown $k$, and hence the difficulty of factoring $n$ is equivalent to that of finding $k$. Clearly, an exhaustive search for $p+q$ based on the equation (11) will require

$$
O\left(2^{|p+q|-|r|}\right)=O\left(2^{\left|\left(p^{\prime}+q^{\prime}\right) / 2 f\right|}\right)
$$

steps. This seems to be the basis for the choice of security parameters in most schemes using a prime order subgroup of $Z_{n}^{*} 8911,13$. However, there exists a much more efficient attack only requiring the square root of the above complexity.

Combining (9) and (11), we have

$$
n+1-(n+1 \bmod r)=\phi(n)+k r .
$$

Since $u^{\phi(n)}=1(\bmod n)$ for an arbitrary $u$ in $Z_{n}^{*}$, raising $u$ to the both sides of (13) yields

$$
u^{n+1-(n+1 \bmod r)}=w^{k}(\bmod n),
$$

where $w=u^{r} \bmod n$ is known. Here we may assume $u$ to have the maximum order $\lambda(n)=2 f p^{\prime} q^{\prime}$ since most elements in $Z_{n}^{*}$ will have this order. Note by symmetry for $p>q$ (hence $p^{\prime}>q^{\prime}$ ) and $q^{\prime}>3$, we have

$$
\operatorname{ord}_{n}(u)=2 f p^{\prime} q^{\prime}>2 f p^{\prime} 3=3(p-1)>2 p>p+q>k r .
$$

So the order of $u$ (greatly) exceeds $k r$ and this means in the transformation from (9), (11) to (14), the quantity $k$ will not be reduced in modulo $\operatorname{ord}_{n}(u)$. 
A straightforward way to solve the equation (14) is to use Shanks' "baby-step giant-step" method (e.g., see [185]). It requires

$$
O\left(2^{|k| / 2}\right)=O\left(2^{(|p+q|-|r|) / 2}\right)
$$

steps of group computation (multiplication modulo $n$ ) and the same order of memory. This is a much lowered time complexity than that in (12) as it is the positive square root of (12)1 However, since space is usually more expensive than time, the large space needed makes this method likely to be infeasible for $k$ with critical sizes. Fortunately there are two memoryless variants of Shanks' method due to Pollard: the rho method and the lambda method [14] (see also 18). Both methods have the same square-root running time, but the space requirement is negligible. Pollard's rho method requires explicit knowledge of the order of the underlying group (i.e., the order of $w$ in (14)), so it can't be used for our purpose. However, the lambda method works even if the group order is not known; The method may produce an exponent with a small multiple of the group order added (modulo addition/subtraction). This is usually not a problem. In particular, for $k$ with sizes of our interest, the order of $w\left(\operatorname{ord}_{n}(w)=p^{\prime} q^{\prime}\right)$ should be much larger than $k$. So we can extract the exact value of $k$ from the lambda method.

In the above we have proven the following statement:

Proposition 2. Let $n=p q$ for $p, q$ being two distinct primes and suppose that $|p| \approx|q|$. When $r$ is a known factor of $(p-1)(q-1)$, then $n$ can be factored in time $O\left(2^{(|n|-2|r|) / 4}\right)$ using Pollard's lambda method.

Most schemes using the key setting in (7) base their security on both the difficulty of factoring $n$ and the difficulty of finding discrete logarithms mod $f$ for the known order $f$. Let us consider minimal requirements for choosing key parameters for such applications. For this, suppose that the current (perhaps not long-term) accepted comfortable margin for computational infeasibility is about $2^{70}$. First of all, the disclosed order $f$ should be at least 140 bits long to thwart Pollard's rho method for finding discrete logarithms mod $f$. Next, Proposition 2 requires that $|n|-2|r|$ should be at least 280 . Therefore, the modulus $n$ should be at least 844 bits long (i.e., $|p|=|q|=422$ ), since $|r|=2|f|+2=282$. More generally, to guarantee the security level of $2^{|f| / 2}$ steps for both the above two attacks, we should have at least

$$
|p|=|q| \geq 3|f|
$$

Note however that though Proposition 2 is the best result currently known (at least to the authors) on exploitation of computing small discrete logarithms, we can't exclude the possibility of existing a more efficient specialized factoring attack for such a key setting.

\footnotetext{
${ }^{1}$ A similar (less general) method for the square-root reduction was first given in [12].
} 


\begin{tabular}{|c||c|c|c|}
\hline$|f|$ & $|k|$ & complexity & actual timing \\
\hline \hline 160 & 31 & $2^{15}$ & 12 seconds \\
\hline 156 & 39 & $2^{20}$ & 197 seconds \\
\hline 152 & 47 & $2^{24}$ & 54 minutes \\
\hline 148 & 55 & $2^{28}$ & 18 hrs 22 min \\
\hline
\end{tabular}

Table 1. Implementation results of Pollard's lambda method for $|n|=704$

From the above analysis we know that 840 should be the least length setting for a modulus $n$ in the construction (7) of which a subgroup of order $f$ is disclosed. Any such moduli with length less than 800 bits are likely to be dangerous. For instance, for an 800-bit $n=p q$ with $p, q$ of similar lengths and for $f$ being a 160 -bit prime, $n$ can be factored in roughly $2^{40}$ steps of multiplication, well within the grasp of a determined attacker. In the case of key setting in 89 $(|f|=140,|p|=|q|=350)$, the cost for factoring is about $2^{35}$ steps. The key setting in Sect.5 of [11] consists of $|f|=160,|p|=|q|=395$, and thus $n$ can be factored in about $2^{38}$ steps.

A prime order subgroup used in Sect.4 of [11] uses the following construction for modulus $n$

$$
n=p q, p=2 p^{\prime} \gamma^{d} f+1, q=2 q^{\prime} f+1,
$$

where $\operatorname{gcd}(\gamma, x)=1$ for $x=p^{\prime}, q^{\prime}, f$. The numbers $f, \gamma$, and $d$ are disclosed. Further, [1] stipulates that $\gamma$ should be in the following order of magnitude

$$
O\left(\operatorname{pol}_{1}(k)\right)^{O\left(\operatorname{poly}_{2}(k)\right)},
$$

where $\operatorname{poly}_{1}(k)$, $\operatorname{poly}_{2}(k)$ are polynomials in $k=\left|p^{\prime}\right|=\left|q^{\prime}\right|$ (this definition of $k$ is specified in Section 2 of [19]). In 11], no information is given on the sizes of $p$ and $q$ with respect to the size of $\gamma^{d}$. However, from the specified order of magnitude, the size of $\gamma^{d}$ is comparable to $\left|p^{\prime}\right|,\left|q^{\prime}\right|$ and it may easily reach a few hundred bits. Note that in such a setting, $4 f^{2} \gamma^{d}$ is a known factor of $(p-1)(q-1)$. So using this factor in the complexity bound in Proposition 2, the time for factoring $n$ can further be lowered by $2^{\left|\gamma^{d}\right| / 2}$ times from the case where only $f$ is disclosed. The only way to avoid factorization is to increase the size of the modulus (and must at the same time limit the degree of $\operatorname{poly}_{2}(k)$ in comparison to that of $\left.\operatorname{poly}_{1}(k)\right)$. Such moduli are likely to exceed a practical size.

We have implemented Pollard's lambda method for solving the equation (14) to verify that our attack actually works. A number of moduli with the structure of (7) have been tested and successfully factored. As nontrivial examples (listed in A), each modulus $n$ has 704 bits and $\phi(n)$ has a factor $r=4 f^{2}$ with $f$ being prime. We computed the exponent $k$ in (14) using our implementation of the lambda method and knowledge of $n$ and $f$ only. Table 1 lists the result of factoring four 704-bit moduli $n$ with $|f|=160,156,152$ and 148, corresponding to $|k|=31,39,47$ and 55, respectively, on a Pentium II PC (Windows 95, 266 
MHz)2 We used the crypto-library with partial assembly coding (developed in Future Systems, Inc.), but the lambda algorithm itself was not much optimized.

Note that Pollard's lambda method can be parallelized with a perfect linear speedup ( $m$-fold speedup with $m$ processors) analogously to the rho method [17]. Exploiting the parallelizability, a $2^{40}$ level time complexity (exceeding the problems in 8911]) can be handled by amateur attackers. For example, using 128 processors, each at the level of $266 \mathrm{MHz}$ Pentium II, we can deduce from our experiment that a 704-bit modulus with $|f|=140$ (resulting time complexity: $2^{35}$ ) can be factored within one day.

\section{Conclusion}

We have analyzed a number of failures in cryptosystems and protocols that use prime order subgroups of $Z_{n}^{*}$, and shown that great care must be taken for such uses. The results of the open trapdoor revealed in Section 2, and the much lowered complexity for factoring $n$ shown in Section 4, may not be new in mathematics; nevertheless to our belief, should be aware to the community of cryptosystem and protocol design.

\section{Acknowledgments}

We are grateful to Colin Boyd of Queensland University of Technology, Brisbane, Liqun Chen, Kenneth Paterson and Nigel Smart of HP Labs., Bristol for their constructive and critical comments. The implementation of lambda algorithm was done by Hyo Sun Hwang of Future Systems, Inc., Seoul. We would like to express special thanks for his time and effort.

\section{References}

1. Bao, F., R. Deng and W. Mao. Efficient and practical fair exchange protocols with off-line TTP. 1998 IEEE Symposium on Security and Privacy. Oakland, May 1998. pages 77-85. IEEE Compute Society.

2. Boyd, C. Digital signature and public key cryptosystem in a prime order subgroup of $Z_{n}^{*}$. First International Conference on Information and Communications Security, ICICS'97 (LNCS 1334), pages 346-355. Springer, 1997.

3. Boyd, C. Presentation in First International Conference on Information and Communications Security, ICICS'97 for fixing a flaw in the paper "Digital signature and public key cryptosystem in a prime order subgroup of $Z_{n}^{*}$ ". Beijing, November 1997.

4. Boyd, C. Personal communications.

5. H. Cohen. A Course in Computational Algebraic Number Theory. Springer-Verlag Graduate Texts in Mathematics 138. 1993.

${ }^{2}$ Actual timings may vary from instance to instance, but the presented timings were obtained from a single run for each instance. 
6. ElGamal, T. A public-key cryptosystem and a signature scheme based on discrete logarithms. IEEE Transactions on Information Theory, IT-31(4):469-472, July 1985.

7. Fiat, A. and A. Shamir. How to prove yourself: Practical solution to identification and signature problems. Advances in Cryptology - Proceedings of CRYPTO'86 (LNCS 263), pages 186-194. Springer-Verlag, 1987.

8. Girault, M. An identity-based identification scheme based on discrete logarithms modulo a composite number. In Advances in Cryptology - Proceedings of EUROCRYPT'90 (LNCS 473), pages 481-486. Springer-Verlag, 1991.

9. Girault, M. and J.C. Paillès. An identity-based scheme providing zero-knowledge authentication and authenticated key-exchange. First European Symposium on Research in Computer Security-ESORICS'90, pages 173-184. 1990.

10. Guillou, L.C. and J.-J. Quisquater. A practical zero-knowledge protocol fitted to security microprocessor minimizing both transmission and memory. In Advances in Cryptology - Proceedings of EUROCRYPT'88 (LNCS 330), pages 123-128. Springer-Verlag, 1988.

11. Kim, S. J., S. J. Park and D. H. Won. Convertible group signatures. Advances in Cryptology - Proceedings of ASIACRYPT'96 (LNCS 1163), pages 310-321. Springer, 1996.

12. Lim, C.H. and P.J. Lee. Sparse RSA secret keys and their generation. Proc. 3rd Workshop on Selected Areas of Cryptography, Aug.15-16, 1996, pp.117-131, Queen's University, Ontario.

13. Park, S., S. Kim and D. Won. ID-based group signature. Electronics Letters. Vol.33, No.19, pages 1616-1617. September 1997.

14. Pollard, J.M. Monte Carlo method for index computation $(\bmod p)$, Mth. Comp., Vol.32, No.143 (1978), pages 918-924.

15. Rabin, M.O. Digital signatures and public-key functions as intractable as factorization. MIT Laboratory for Computer Science, Technical Report, MIT/LCS/TR-212. 1979.

16. Rivest, R.L., A. Shamir and L.M. Adleman. A method for obtaining digital signatures and public-key cryptosystems. Communications of the $A C M \mathrm{v} .21, \mathrm{n} .2$, pages 120-126. 1978.

17. Van Oorschot, P.C. and M.J. Wiener, Parallel collision search with application to hash functions and discrete logarithms, Proc. 2nd ACM Conference on Computer and Communications Security, Nov.2-4 1994, Fairfax, Verginia, pp.210-218. (a revised version to appear in Journal of Cryptology).

18. Van Oorschot, P.C. and M.J. Wiener, On Diffie-Hellman key agreement with short exponents, Advances in Cryptology-EUROCRYPT'96 (LNCS 1070), pages 332343, Springer-Verlag, 1996.

19. Zheng, Y., T. Matsumoto and H. Imai. Residuosity problem and its applications to cryptography. Trans. IEICE, Vol.E71, No.8, pages 759-767. 1988. 


\section{A Factorization Examples}

Listed here are four examples of factoring composite numbers $n_{i}=p_{i} q_{i}$ with $\left|n_{i}\right|=704(i=1,2,3,4)$. Each of them is in the structure of $(7)$ with a disclosed prime number $f_{i}$ satisfying $4 f_{i}^{2} \mid \phi\left(n_{i}\right)$, respectively. (Each disclosed number is the prime order of a subgroup in $Z_{n_{i}}^{*}$.) The factorization procedure, which uses knowledge of $n_{i}$ and $f_{i}$ only, consists of the following steps. Let $r_{i}=4 f_{i}^{2}$; find $k_{i}$ by solving (14) using Pollard's lambda method and an arbitrary $u \in Z_{n_{i}}^{*}$; finally compute the sum of the two factors $p_{i}+q_{i}$ from (11).

$n_{1}=$

44038789839272366089711678758549018585781306376481219309675016031571 59110337100473118835059630202675486525459776285546455730190806180736 80685283791730902303592537905553614145315768184318908979789396252844 29109969

$f_{1}=1279307573885884659565630730667563032209304623881\left(\left|f_{1}\right|=160\right)$

$k_{1}=2027611962\left(\left|k_{1}\right|=31\right)$

$p_{1}+q_{1}=$

13273784976299044547490668423368063756808688033061030560778659385613 470153995077873141935147799796097271326

$p_{1}=$

67346271288005758035121802466486698270341935982350484466308017371045 48023040804821535454700449823437654803

$q_{1}=$

65391578474984687439784881767193939297744944348259821141478576485089 22130954273051606480447349972659616523

$n_{2}=$

64554109864989573045209981572033362472631867814455170482343370081941 75374614719836519737903434764927667241348701311953011118637118842580 20822822610693337656026764057069349846323931678811070617670913443882 33210497

$f_{2}=86377634978865770214549290023394612171958862633\left(\left|f_{2}\right|=156\right)$

$k_{2}=538747074867\left(\left|k_{2}\right|=39\right)$

$p_{2}+q_{2}=$

16078574203113175306560234663909305050475184101648412171345388343619 358401841450007235060358847689467446062

$p_{2}=$

77635567327820418210972396543355782102974407297844441005838281962192 92538274120851161987346342157066235939

$q_{2}=$

83150174703311334854629950095737268401777433718639680707615601474000 65863567329156073073012505532401210123

$n_{3}=$

49717795134695045326475112627330486057331272421904875018507553084533 
28535166549501577613963887317483273134902374557567913651145723535990 21740913276114932911412468599093017719864542252814715746579589808031 33009497

$f_{3}=5199318848515295611566769410707286918384452513\left(\left|f_{3}\right|=152\right)$

$k_{3}=130766945849270\left(\left|k_{3}\right|=47\right)$

$p_{3}+q_{3}=$

14140047706411940330625844939512048374565564888066290058212225984441 079384020823262406209041853117141243942

$p_{3}=$

65528757600968992834709152083734731421153187063781982357964242382851 02130907506525723131350775223761127159

$q_{3}=$

75871719463150410471549297311385752324502461816880918224158017461559 77253113316736683077691077893380116783

$n_{4}=$

48674186760539467681018145523056045973888236415345231292673943613500 03686092964604087243177579323851165956239195451395380585901768379305 01449596943339226807057417747240009896818786895758692021292580430607 40210629

$f_{4}=330123952043083490387386128078634198300183001\left(\left|f_{4}\right|=148\right)$

$k_{4}=32139335064789130\left(\left|k_{4}\right|=55\right)$

$p_{4}+q_{4}=$

14010413393076943015390974069221998326136511372422548051245305917863 342832550005586489706641326886563369946

$p_{4}=$

76366606542329158917195431596702997461688808846138079970516645219141 51997938395920083821905816573720062483

$q_{4}=$

63737527388440271236714309095516985799676304878087400541936413959491 90834611609666405884735510312843307463 